\title{
Release Pattern of Salivary Chromogranin A in Pediatric Subjects with Obstructive Sleep Apnea
}

\author{
Heung-Ku Lee, MD, Hye-Lim Son, MD, Soo-Hyung Lee, MD, Chan-Soon Park, MD, PhD \\ Department of Otolaryngology Head and Neck Surgery, St. Vincent's Hospital, The Catholic University of Korea College of Medicine, Suwon, Korea
}

Background and Objective Sleep-disordered breathing (SDB) is associated with activation of the stress response, including the autonomic nervous system. Salivary chromogranin A ( $\mathrm{sCgA})$ is considered a valuable indicator of sympathoadrenal activity. We examined the relationship between sCgA and polysomnography (PSG) parameters.

Methods In this prospective study, we enrolled 103 children who underwent a physical examination and fully attended in-lab PSG. Saliva was collected at night before PSG and in the early morning after PSG.

Results The subjects $(\mathrm{n}=103)$ were divided into control $[\mathrm{n}=41$, apnea-hypopnea index $(\mathrm{AHI}) \leq 1]$ and obstructive sleep apnea syndrome (OSAS; $\mathrm{n}=62$, AHI $>1$ ) groups. The OSAS group was subdivided into mild $(1<\mathrm{AHI} \leq 5)$, moderate $(5<\mathrm{AHI} \leq 10)$, and severe $(10<\mathrm{AHI})$ groups. There was no significant difference in the sCgA parameters between the control and OSAS groups. No significant difference was observed in SCgA parameters between the control group and OSAS subgroups (mild, moderate, and severe). No circadian rhythm was detected in $\mathrm{SCgA}$ secretion, and no difference in sCgA concentrations was measured at the two time points.

Conclusions Our findings suggest that sCgA secretion was not influenced by OSAS severity and no definitive circadian rhythm was detected in pediatric subjects. Further study is needed to establish whether there is a circadian rhythm in pediatric subjects.

Sleep Med Res 2014;5(2):62-66

Key Words Child, Chromogranin A, Saliva, Sleep apnea.

Received: November 10, 2014

Revised: January 13, 2015

Accepted: February 3, 2015

Correspondence

Chan-Soon Park, MD, PhD

Department of Otolaryngology-HNS,

St. Vincent's Hospital,

The Catholic University of Korea

College of Medicine, 93 Jungbu-daero,

Paldal-gu, Suwon 442-723, Korea

Tel +82-31-249-8304

Fax +82-31-257-3752

E-mail pcs0112@catholic.ac.kr

\section{INTRODUCTION}

Sleep-disordered breathing (SDB) is a common condition in the pediatric population with a prevalence of $0.7-13.0 \% .{ }^{1}$ Obstructive sleep apnea syndrome (OSAS) is characterized by episodes of partial or complete obstruction of the upper airways during sleep. Recurrent upper airway obstruction results in hypoxemia, hypercapnia, and significant swings in intrathoracic pressure. Such alterations drive the autonomic nervous system (ANS) towards increased basal tone and reactivity of the sympathetic nervous system (SNS). ${ }^{2}$

Various non-invasive and indirect methods have been used to evaluate the ANS in children, because application of invasive and direct methods in this group is difficult. Among these probes, recently, and salivary biomarkers have been used to demonstrate ANS alterations in children. Sampling of saliva has many advantages in children, because it is an easy, safe, and pain-free method to collect and easy to conduct multiple samplings even in children.

Park et al. ${ }^{3,4}$ reported salivary cortisol and alpha amylase was associated with OSAS severity in pediatric subjects. Especially, the measurement of salivary cortisol levels before and after adenotonsillectomy may predict the outcome of adenotonsillectomy as a treatment for OSAS in pediatric subjects. $^{5}$

Salivary chromogranin A ( $\mathrm{sCgA}$ ) has been suggested as another biomarker of sympathoadrenal activity and it reflects psychological stress. ${ }^{6}$ Chromogranin A (CgA) is a $48-\mathrm{kDa}$ acidic glycoprotein that is released along with catecholamines from the adrenal medulla and sympathetic nerve endings. ${ }^{7}$ Thus, it is considered to be a valuable indicator of symphaoadrenal activity. Re- 
cently, sCgA was shown to be produced by the human submandibular gland and secreted into the saliva. Den et al. ${ }^{8}$ reported a circadian rhythm of $\mathrm{sCgA}$ in humans. They reported that the sCgA levels peaked at awakening and then quickly decreased to a nadir 1 hour later, remaining at a low level throughout the day, and finally increasing again at night.

Lee et al. ${ }^{9}$ suggested that $\mathrm{sCgA}$ was a useful marker of stress. They examined sCgA responses before and after venipuncture in hospitalized children. sCgA levels immediately after venipuncture were significantly higher than those before it.

Our previous data showed that salivary biomarkers, including salivary cortisol and alpha amylase, were correlated with OSA and may be useful for predicting SDB severity in pediatric subjects. To our knowledge, there are few reported studies of sCgA in OSAS patients. Thus, we investigated the association of sCgA as a biomarker of the SNS in pediatric subjects with OSAS.

\section{METHODS}

\section{Subjects}

In total, 103 children, aged 3-13 years, with suspicion of SDB or enlarged tonsils/adenoids regardless of SDB and who presented at the Department of Otolaryngology Head and Neck Surgery, St. Vincent's Hospital, Suwon, Republic of Korea, between July 2011 and June 2012 were enrolled prospectively in this study.

After taking a history from the parents regarding past medical conditions and medications and performing a complete otolaryngological examination, fully attended overnight polysomnography (PSG) was performed, and saliva from each child was collected in special tubes at $\sim 10$ pm before PSG and the morning following PSG. We excluded subjects with other chronic medical or psychiatric illnesses, sinonasal disease (antrochoanal polyp or nasal polyposis), and congenital anomalies with craniofacial abnormalities.

The objectives and methods of the study were explained to all participants and their parents. Informed consent was obtained from the parents before enrollment. This study protocol was reviewed and approved by the Ethics Committee for Clinical Studies at St. Vincent's Hospital, The Catholic University of Korea (VC11TISI0090).

\section{Polysomnography}

Fully attended overnight PSG was performed on all participants using a computerized PSG system (Somnologica software and Embla S700/A10 hardware; Embla Systems Inc., Broomfield, CO, USA). Physiological signals were recorded from four electroencephalogrphy (EEG) channels, two electrooculography channels, one electrocardiography-lead, three electromyograms (chin, anterior tibialis muscles, right and left), and one body position sensor. Respiratory signals were monitored with a nasal pressure transducer, an oral thermistor, a thoracic and abdominal re- spiratory effort sensor (piezo type), a pulse oxymeter, and a neck vibration sensor for snoring. All parameters were interpreted manually by a technician and reviewed by a physician.

Apnea was defined as the absence or reduction of $>90 \%$ of oronasal airflow for $>90 \%$ of the entire event for a period lasting at least two regular breaths. Hypopnea was defined as a $50 \%$ or greater air flow reduction lasting for at least two regular breaths, with desaturation of $3 \%$ or greater, awakening, or EEG arousal. The apnea-hypopnea index (AHI) was defined as the number of apnea and hypopnea episodes per hour of total sleep time. OSA in pediatric subjects was defined as $\mathrm{AHI}>1$.

\section{Measurement of sCgA}

We instructed the children's parents to avoid extraordinary exercise on the day of the PSG test before saliva sampling. To minimize error, the children rinsed their mouth with water within 10 minutes before saliva sampling. We avoided sampling saliva collected within 60 minutes after eating a major meal, according to the manufacturer's protocol.

Saliva samples were collected using an unstimulated method that included tilting the head forward, allowing the saliva to pool on the floor of the mouth, and passing the saliva through a short straw into a polypropylene vial. Two saliva samples were collected in special tubes to check the diurnal variation of salivary alpha amylase (sAA): at $\sim 10 \mathrm{pm}$ on the day before PSG and at 7 am on the morning following PSG (within 60 minutes of waking up). The samples were refrigerated within 30 minutes and frozen at $-20^{\circ} \mathrm{C}$ within 4 hours after collection. The samples were thawed completely on the day of the assay, vortexed, and centrifuged $(1500 \times \mathrm{g}, 15$ minutes). Samples were kept at room temperature before transfer to the assay plate. We measured sAA activity using a commercially available kinetic reaction assay kit (Salimetrics, State College, PA, USA).

The saliva parameters were divided as follows: 1) 'night' was the sCgA value before PSG (n-sCgA), 2) 'morning' was the sCgA value after PSG (m-sCgA), 3) 'subtracted' was defined as subtracting the night measurement from the morning measurement (sub-sCgA), and 4) the 'ratio' was the sCgA ratio of the morning and night measurements ( $\mathrm{r}-\mathrm{sCgA}$ ).

\section{Statistics}

Statistical analyses were conducted using the SPSS software (SPSS Inc., Chicago, IL, USA). Quantitative variables in the OSAS $(\mathrm{AHI}>1)$ and control $(\mathrm{AHI} \leq 1)$ groups were compared using Student's t-test, Fisher's exact test, and the $\chi^{2}$ test. Analysis of variance test were performed to investigate the relationship among the control group and each OSAS subgroup. Paired t-tests were performed to assess the circadian rhythm of sCgA. A value of $\mathrm{p}<$ 0.05 was taken to indicate statistical significance. 
Table 1. Basic demographic, anthropometric, and conventional polysomnographic date

\begin{tabular}{|c|c|c|c|c|}
\hline & & Control $(n=41)$ & OSAS $(n=62)$ & $\mathrm{p}$ value \\
\hline \multirow[t]{2}{*}{ Gender } & Male (\%) & $19(46.3)$ & $44(71.0)$ & 0.012 \\
\hline & Female (\%) & $22(53.7)$ & $18(29.0)$ & \\
\hline Age & & $7.4 \pm 2.9$ & $6.8 \pm 3.1$ & 0.310 \\
\hline BMI & & $18.1 \pm 3.0$ & $18.3 \pm 3.9$ & 0.715 \\
\hline \multirow[t]{3}{*}{ Sleep parameters } & Total sleep time (min) & $471.5 \pm 41.2$ & $466.7 \pm 38.4$ & 0.545 \\
\hline & Sleep latency (min) & $13.9 \pm 11.4$ & $18.0 \pm 16.2$ & 0.140 \\
\hline & Sleep efficiency (\%) & $93.6 \pm 6.4$ & $91.7 \pm 7.1$ & 0.184 \\
\hline \multirow{4}{*}{$\begin{array}{l}\text { Sleep stage duration } \\
\text { (\% of total sleep time) }\end{array}$} & N1 & $5.2 \pm 2.6$ & $8.2 \pm 4.9$ & $<0.001$ \\
\hline & $\mathrm{N} 2$ & $47.6 \pm 5.6$ & $45.2 \pm 6.5$ & 0.054 \\
\hline & N3 & $27.1 \pm 5.2$ & $28.1 \pm 6.4$ & 0.426 \\
\hline & REM & $20.0 \pm 4.2$ & $18.7 \pm 5.5$ & 0.191 \\
\hline \multirow[t]{2}{*}{ Respiratory parameters } & AHI & $0.2 \pm 0.3$ & $13.6 \pm 19.4$ & $<0.001$ \\
\hline & Lowest $\mathrm{O}_{2}$ saturation & $90.5 \pm 15.2$ & $89.7 \pm 5.9$ & 0.770 \\
\hline
\end{tabular}

Data are presented as means $\pm \mathrm{SD}$, and as $\mathrm{n}(\%)$.

$P$ values of difference by $\chi^{2}$ test, Fisher's exact test, and Student's t-test (two-tailed) with statistical significance set at $\alpha=0.05$.

AHI: apnea-hypopnea index, BMI: body mass index, OSAS: obstructive sleep apnea syndrome, REM: rapid eye movement, SD: standard deviation.

Table 2. Association between the normal group and the OSAS group

\begin{tabular}{lccc}
\hline $\mathrm{sCgA}$ & Control $(\mathrm{n}=41)$ & OSAS $(\mathrm{n}=62)$ & $\mathrm{p}$ value \\
\hline $\mathrm{n}$-sCgA & $12.1 \pm 14.6$ & $10.6 \pm 14.0$ & 0.619 \\
$\mathrm{~m}-\mathrm{sCgA}$ & $10.3 \pm 11.5$ & $10.0 \pm 12.4$ & 0.904 \\
$\mathrm{sub}-\mathrm{sCgA}$ & $1.8 \pm 15.1$ & $0.5 \pm 18.2$ & 0.739 \\
$\mathrm{r}-\mathrm{sCgA}$ & $3.4 \pm 9.5$ & $5.2 \pm 11.0$ & 0.439 \\
\hline
\end{tabular}

P values of difference between control vs. OSAS by Student's t-test. OSAS: obstructive sleep apnea syndrome, sCgA: salivary chromogranin A, n-sCgA: the sCgA value before PSG, m-sCgA: the sCgA value after PSG, sub-sCgA: subtracting the night measurement from the morning measurement, $\mathrm{r}$-sCgA: the ratio of morning and night measurements, PSG: polysomnography.

\section{RESULTS}

The subjects $(\mathrm{n}=103)$ were divided into control $(\mathrm{n}=41$, AHI $<1)$ and OSAS $(n=62, A H I \geq 1)$ groups. Demographic data and PSG findings are presented in Table 1. The ratio of males:females in the OSAS group [44 males (71.0\%), 18 females (29.0\%)] differed from that in the control group [19 males (46.3\%), 22 females (53.7\%)]. No significant difference was observed between the two groups in age or body mass index. None of the sleep parameters was significantly different between the OSAS and control groups. N1 duration was significantly longer in the OSAS group ( $<<0.001$ ), but N2 duration, slow wave sleep (N3), and rapid eye movement duration showed no significant difference. The lowest oxygen saturation in the OSAS group was not different from the control group. The number of AHI events per hour was significantly higher in the OSAS group than in the control group (Table 1).

There were no significant differences in $\mathrm{sCgA}$ parameters be- tween the control and OSAS groups (Table 2).

After dividing the OSAS group into mild $(1<\mathrm{AHI} \leq 5)$, moderate $(5<\mathrm{AHI} \leq 10)$, and severe $(\mathrm{AHI}>10)$ subgroups, sCgA parameters showed no significant difference between the control and any OSAS subgroup (Table 3). There was no significant difference between the morning and night sCgA levels in each subgroup and the total group (Table 4).

\section{DISCUSSION}

We investigated the levels of sCgA in response to OSAS in pediatric subjects. In our results, $\mathrm{sCgA}$ showed no significant difference between the control and OSAS groups, and showed no significant difference according to OSAS severity.

Chromogranin A is soluble protein, and its concentration is considered to be a surrogate for catecholamine because CgA and catecholamines are co-released into the extracellular environment. Dimsdale et al..$^{10}$ reported that the plasma CgA level correlated with the norepinephrine release rate. They suggested that CgA may offer a novel perspective on peripheral sympathetic activity.

Obstructive sleep apnea syndrome is associated with various cardiovascular morbidities in pediatric subjects. Many studies have reported cardiovascular sequelae, including changes in heart rate, blood pressure, and cardiac morphology in children with OSAS. ${ }^{2,11-13}$ As mentioned above, the underlying mechanism for the cardiovascular complications is thought to be changes in the ANS. ${ }^{14}$ Thus, we expected that sCgA may be increased in the morning and according to the OSAS severity. However, in contrast to our expectations, sCgA parameters showed no significant 
Table 3. Association between the normal and OSAS groups

\begin{tabular}{|c|c|c|c|c|c|}
\hline sCgA & $\begin{array}{c}\text { Control, } \\
0 \leq \mathrm{AHI} \leq 1(\mathrm{n}=41)\end{array}$ & $\begin{array}{c}\text { Mild, } \\
1<\text { AHI }<5(\mathrm{n}=25)\end{array}$ & $\begin{array}{c}\text { Moderate } \\
5 \leq \mathrm{AHI}<10(\mathrm{n}=12)\end{array}$ & $\begin{array}{c}\text { Severe, } \\
10 \leq \text { AHI }(\mathrm{n}=25)\end{array}$ & $\mathrm{p}$ value \\
\hline n-sCgA & $12.1 \pm 14.6$ & $10.1 \pm 13.5$ & $15.0 \pm 19.6$ & $8.7 \pm 10.9$ & 0.672 \\
\hline m-sCgA & $10.3 \pm 11.5$ & $8.7 \pm 11.8$ & $13.6 \pm 18.1$ & $9.7 \pm 9.3$ & 0.760 \\
\hline sub-sCgA & $11.2 \pm 10.8$ & $8.6 \pm 8.1$ & $14.3 \pm 15.6$ & $9.2 \pm 6.7$ & 0.434 \\
\hline r-sCgA & $1.8 \pm 15.1$ & $1.4 \pm 19.7$ & $1.4 \pm 21.2$ & $-1.0 \pm 15.3$ & 0.953 \\
\hline
\end{tabular}

P values of difference between normal vs. mild vs. moderate vs. severe by ANOVA test.

OSAS: obstructive sleep apnea syndrome, sCgA: salivary chromogranin A, n-sCgA: the sCgA value before PSG, m-sCgA: the sCgA value after PSG, sub-sCgA: subtracting the night measurement from the morning measurement, $r$-sCgA: the ratio of morning and night measurements, AHI: apnea-hypopnea index, ANOVA: analysis of variance, PSG: polysomnography.

Table 4. Association between chromo-night and chromo-morning in each group

\begin{tabular}{lccc}
\hline \multicolumn{1}{c}{ Group } & $\mathrm{n}$-sCgA & $\mathrm{m}$-sCgA & $\mathrm{p}$ value \\
\hline Total $(\mathrm{n}=103)$ & $11.2 \pm 14.2$ & $10.2 \pm 12.0$ & 0.557 \\
Control $(\mathrm{n}=41)$ & $12.1 \pm 14.6$ & $10.3 \pm 11.5$ & 0.479 \\
OSAS $(\mathrm{n}=62)$ & $10.6 \pm 14.0$ & $10.0 \pm 12.4$ & 0.834 \\
\hline
\end{tabular}

$\mathrm{P}$ values of difference by paired t-test.

sCgA: salivary chromogranin $\mathrm{A}, \mathrm{n}-\mathrm{sCgA}$ : the sCgA value before PSG, m-sCgA: the sCgA value after PSG, OSAS: obstructive sleep apnea syndrome, PSG: polysomnography.

difference, which might have been due to the narrow range of $\mathrm{AHI}$ in pediatrics. Pediatric OSAS may be diagnosed if $\mathrm{AHI}$ is more than 1 and severe OSAS in children if AHI is more than 10. However, adult OSAS has a wide range of AHI and desaturation. In pediatric OSAS, the relatively small range of $\mathrm{AHI}$ and desaturation may not be enough to cause a change in $\mathrm{sCgA}$. To our knowledge, there is no other report on sCgA in adult OSAS. Further studies are needed to make clarify the situation in adults.

To date, CgA has been investigated as a sensitive marker for the diagnosis of endocrine tumors, such as pheochromocytoma. ${ }^{15}$ Recently, there have been many studies about CgA as an indicator of psychological stress and chronic diseases. Nakane et al. ${ }^{16}$ reported that sCgA levels were elevated immediately before subjects gave an oral presentation and then decreased immediately after the presentation was finished, while it was not significantly elevated after physical exercise.

In an adult study, Den et al. ${ }^{17}$ reported that the awakening sCgA concentration was decreased in the severe depression subscale. This study suggested that chronic psychological stress may influence sCgA secretion via chronic stress-related attenuation of the activity of the sympathetic-adrenomedullary system in healthy adult males. Reshma et al. ${ }^{18}$ reported that the levels of CgA in the plasma and saliva were elevated in subjects with stress-induced chronic periodontitis. There were inconsistent results of sCgA secretion in response to chronic stress.

As mentioned above, sCgA has been reported to show a circadian rhythm in adults that peaked at awakening and then quickly decreased to a nadir 1 hour later, remaining at a low level throughout the day, and finally increasing again at night. However, plasma CgA did not show any obvious circadian rhythm, which might suggest salivary and plasma CgA have different routes of secretion. ${ }^{8}$ Hong et al. ${ }^{19}$ reported a similar circadian pattern of sCgA in adult women. They recommended that appropriate sampling times for the salivary stress markers, CgA and cortisol, were at 7:00 (time of awakening), 8:00 (1 hour after awakening), 17:30 (early evening), and 22:30 (before sleep) for job stress assessments.

However, to our knowledge, there is no report related to chronic stress and $\mathrm{sCgA}$ secretion in pediatric subjects. Our results suggest that chronic stress in pediatric subjects may have little effect on sCgA. Further studies are required to assess $\mathrm{sCgA}$ response at awakening in pediatric subjects.

In our study, we performed sampling at two time points (time of awakening and before sleep). According to studies on sAA, the diurnal slope of sAA might be an alternative analysis strategy for assessing altered ANS function. ${ }^{20}$ A study by Out et al., ${ }^{21}$ which compared the distributions of each of the diurnal slopes of sAA to the one based on four time points (waking, 12:00 pm, 5:00 pm, and 9:00 pm) suggested that the collection of a morning (waking) and an evening sample was sufficient to accurately characterize the diurnal slope.

Based on the papers mentioned above, two time point sampling was used in our study. There was no significant difference between morning and night levels of $\mathrm{sCgA}$ in the control or OSAS group. In human adult studies, the level of sCgA has a definite circadian release pattern. This result suggests that the sCgA release pattern in children may differ from that in adults. Because baseline sCgA levels vary among individuals, each subject showed a wide sCgA range. Further study is required to establish whether there is a circadian rhythm in pediatric subjects.

Despite its prospective design, our study had several limitations. First, the control group was not matched for gender, age, or number. Second, the subject's daytime activities were not fully controlled and third, we only used two time points for saliva sampling.

\section{Conclusions}

In our results, $\mathrm{SCgA}$ showed no significant difference between the control and OSAS groups, and showed no significant difference according to OSAS severity. OSAS did not influence the level of sCgA. However, in pediatric subjects, further study is required to determine whether there is a circadian release pattern, 
because circadian rhythm of sCgA in children may differ from that in adults.

\section{Acknowledgments}

Statistical consultation was provided by the Department of Biostatistics of the Catholic Research Coordinating Center.

\section{Conflicts of Interest}

The authors have no financial conflicts of interest.

\section{REFERENCES}

1. Bixler EO, Vgontzas AN, Lin HM, Liao D, Calhoun S, Vela-Bueno A, et al. Sleep disordered breathing in children in a general population sample: prevalence and risk factors. Sleep 2009;32:731-6.

2. Bhattacharjee R, Kheirandish-Gozal L, Pillar G, Gozal D. Cardiovascular complications of obstructive sleep apnea syndrome: evidence from children. Prog Cardiovasc Dis 2009;51:416-33.

3. Park CS, Guilleminault C, Hwang SH, Jeong JH, Park DS, Maeng JH. Correlation of salivary cortisol level with obstructive sleep apnea syndrome in pediatric subjects. Sleep Med 2013;14:978-84.

4. Park CS, Guilleminault C, Park HJ, Cho JH, Lee HK, Son HL, et al. Correlation of salivary alpha amylase level and adenotonsillar hypertrophy with sleep disordered breathing in pediatric subjects. J Clin Sleep Med 2014;10:559-66.

5. Jeong JH, Guilleminault C, Park CS, Son HL, Lee HK, Hwang SH, et al. Changes in salivary cortisol levels in pediatric patients with obstructive sleep apnea syndrome after adenotonsillectomy. Sleep Med 2014;15:672-6.

6. Obayashi K. Salivary mental stress proteins. Clin Chim Acta 2013;425: 196-201.

7. Saruta J, Tsukinoki K, Sasaguri K, Ishii H, Yasuda M, Osamura YR, et al. Expression and localization of chromogranin A gene and protein in human submandibular gland. Cells Tissues Organs 2005;180:237-44.

8. Den R, Toda M, Nagasawa S, Kitamura K, Morimoto K. Circadian rhythm of human salivary chromogranin A. Biomed Res 2007;28:57-60.

9. Lee T, Shimizu T, Iijima M, Obinata K, Yamashiro Y, Nagasawa S. Evaluation of psychosomatic stress in children by measuring salivary chromogranin A. Acta Paediatr 2006;95:935-9.

10. Dimsdale JE, O'Connor DT, Ziegler M, Mills P. Chromogranin A correlates with norepinephrine release rate. Life Sci 1992;51:519-25.

11. Marcus CL, Greene MG, Carroll JL. Blood pressure in children with obstructive sleep apnea. Am J Respir Crit Care Med 1998;157(4 Pt 1): 1098-103.

12. Miman MC, Kirazli T, Ozyurek R. Doppler echocardiography in adenotonsillar hypertrophy. Int J Pediatr Otorhinolaryngol 2000;54:21-6.

13. Ng DK, Wong JC, Chan CH, Leung LC, Leung SY. Ambulatory blood pressure before and after adenotonsillectomy in children with obstructive sleep apnea. Sleep Med 2010;11:721-5.

14. Hakim F, Gozal D, Kheirandish-Gozal L. Sympathetic and catecholaminergic alterations in sleep apnea with particular emphasis on children. Front Neurol 2012;3:7.

15. d'Herbomez M, Do Cao C, Vezzosi D, Borzon-Chasot F, Baudin E; Groupe des tumeurs endocrines (GTE France). Chromogranin A assay in clinical practice. Ann Endocrinol (Paris) 2010;71:274-80.

16. Nakane H, Asami O, Yamada Y, Harada T, Matsui N, Kanno T, et al. Salivary chromogranin A as an index of psychosomatic stress response. Biomed Res 1998;19:401-6.

17. Den R, Toda M, Ohira M, Morimoto K. Levels of awakening salivary $\mathrm{CgA}$ in response to stress in healthy subjects. Environ Health Prev Med 2011;16:155-7.

18. Reshma AP, Arunachalam R, Pillai JK, Kurra SB, Varkey VK, Prince MJ. Chromogranin A: novel biomarker between periodontal disease and psychosocial stress. J Indian Soc Periodontol 2013;17:214-8.

19. Hong RH, Yang YJ, Kim SY, Lee WY, Hong YP. Determination of appropriate sampling time for job stress assessment: the salivary chromogranin A and cortisol in adult females. J Prev Med Public Health 2009; 42:231-6.

20. Nater UM, Rohleder N, Schlotz W, Ehlert U, Kirschbaum C. Determinants of the diurnal course of salivary alpha-amylase. Psychoneuroendocrinology 2007;32:392-401.

21. Out D, Granger DA, Sephton SE, Segerstrom SC. Disentangling sources of individual differences in diurnal salivary a-amylase: reliability, stability and sensitivity to context. Psychoneuroendocrinology 2013;38: $367-75$. 